\title{
A new development in magnetic particle tracking technology and its application in a sheared dense granular flow
}

Cite as: Rev. Sci. Instrum. 90, 065116 (2019); https://doi.org/10.1063/1.5100739

Submitted: 19 April 2019 . Accepted: 01 June 2019 . Published Online: 26 June 2019

Xingtian Tao, Xuemin Tu, and Huixuan Wu
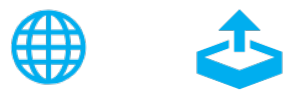

View Online

Export Citation

\section{ARTICLES YOU MAY BE INTERESTED IN}

The translational and rotational motions of a cylindrical particle in a granular shear flow inside a split bottom Couette cell

Physics of Fluids 32, 073310 (2020); https://doi.org/10.1063/5.0015175

A direct comparison of high-speed methods for the numerical Abel transform

Review of Scientific Instruments 90, 065115 (2019); https://doi.org/10.1063/1.5092635

Measuring vorticity vector from the spinning of micro-sized mirror-encapsulated spherical particles in the flow

Review of Scientific Instruments 90, 115111 (2019); https://doi.org/10.1063/1.5121016

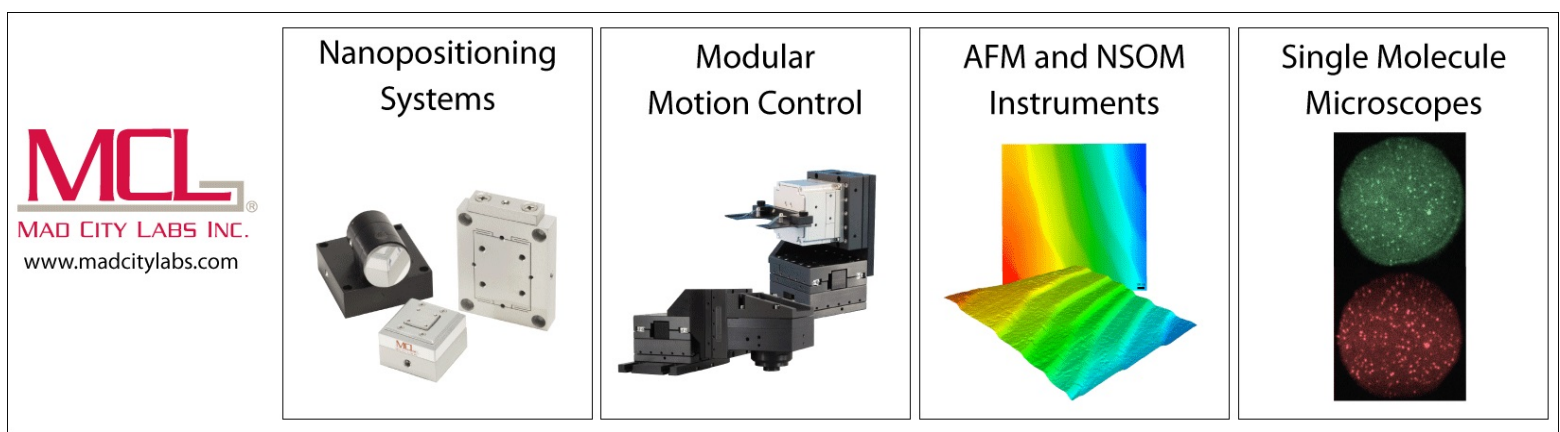




\title{
A new development in magnetic particle tracking technology and its application in a sheared dense granular flow
}

\author{
Cite as: Rev. Sci. Instrum. 90, 065116 (2019); doi: 10.1063/1.5100739 \\ Submitted: 19 April 2019 - Accepted: 1 June 2019• \\ Published Online: 26 June 2019
}

Xingtian Tao, ${ }^{1}$ Xuemin $\mathrm{Tu}^{2}$ and Huixuan $\mathrm{Wu}^{1, \mathrm{a})}$

\author{
AFFILIATIONS \\ ${ }^{1}$ Aerospace Engineering Department, University of Kansas, Lawrence, Kansas 66045, USA \\ ${ }^{2}$ Department of Mathematics, University of Kansas, Lawrence, Kansas 66045, USA \\ a) Email: hwu@ku.edu
}

\begin{abstract}
This paper presents a new development in the magnetic particle tracking (MPT) technology that measures the translational and rotational motions of a small particle. A main advantage of MPT is that it is able to track objects in an opaque environment without using radioactive material or X-rays. In addition, it can provide information about the orientation and rotation of the object, which is difficult to obtain using other technologies. However, the reconstruction process of MPT using standard optimization approaches is very time consuming and, therefore, limits its applications. In this work, two new MPT reconstruction algorithms are examined and the results are compared with the optimization approach. The extended Kalman filter (EKF) algorithm has the same accuracy as the optimization method but is orders of magnitude faster. The speed of the sequential importance sampling approach is between those of the above two methods. The accuracy of position obtained using EKF is about $0.6 \%$, and the uncertainty of orientation is less than $1.5^{\circ}$. The MPT is applied to measure a dense granular shear flow to investigate the spatial distribution of a tracer particle.
\end{abstract}

Published under license by AIP Publishing. https://doi.org/10.1063/1.5100739

\section{INTRODUCTION}

Tracking the motion of an object plays a crucial role in the study of turbulent mixing, granular flow, sedimentation, and cellular biology. ${ }^{1-4}$ Advanced particle tracking technologies are usually optical based (e.g., Lagrangian particle tracking ${ }^{5}$ ), and some algorithms are proposed to improve the accuracy. ${ }^{6,7}$ Their precision is high, but they have limited use in opaque multiphase flows or granular motions, and it takes a lot effort to extract the rotational information of the object. ${ }^{8,9}$ A group of nonintrusive and nonoptical tracking methods have been developed for opaque environments. For instance, radioactive particle tracking (RPT) and positron emission particle tracking (PEPT) are used to obtain the kinetics of particles in a fluidized bed. ${ }^{8-13}$ The RPT directly uses the gamma radiation emitted in the beta-decay of a source particle. The PEPT uses radioactive material such as ${ }^{18} \mathrm{~F},{ }^{61} \mathrm{Cu}$, and ${ }^{66} \mathrm{Ga}$ to label a particle. These materials emit positrons, which annihilate with electrons and generate back-to-back gamma rays. The gamma rays can be monitored by gamma detectors, from which the position of the source can be determined. In addition, X-rays have also been used to measure motion in an opaque environment, such as air bubbles in gas-solid fluidized beds and gas-liquid reactors. ${ }^{14-16}$ Heindel and co-workers have reviewed the X-Ray applications in the visualization of multiphase flows. ${ }^{17,18}$

However, the methods listed above have shortcomings (Table I). The optical methods are not operable in opaque flows. Although the RPT, PEPT, and X-rays can be used, they require expensive equipment and special radioactive material operating expertise. In addition, the RPT and PEPT can only monitor the particle's translation, but not the orientation or rotation, which carries important granular dynamics information. The X-ray tomography is only able to detect the orientation of needlelike objects. The limits of measurement technologies and the lack of experimental results have delayed the development of granular dynamics theory and modeling.

A magnetic particle tracking (MPT) technology ${ }^{20,21}$ has recently been proposed to address the above issues. The MPT was introduced in the medical field for examining the gastrointestinal 
TABLE I. Pros and cons of the widely used particle tracking technologies.

\begin{tabular}{llll}
\hline \hline Method & Optical-based methods & RPT/PEPT/X-rays & \multicolumn{1}{c}{ MPT } \\
\hline Cost & Expensive & Very expensive & Inexpensive \\
Working condition & Transparent only & Transparent or opaque & Transparent or opaque \\
Safety requirement & Laser operation & Radiation protection & None \\
Spatial resolution & High & Relatively low & Relatively low \\
Rotation motion & Difficult & Generally, no & Yes \\
\hline \hline
\end{tabular}

tract without the risk of radiation exposure. ${ }^{22,23}$ In other early efforts, the MPT method was used to track large objects in dense gas fluidized beds with different densities and air flow rates. ${ }^{24-26}$ Recently, Köhler et al. studied the mixing and segregation of fuel in a bubbling fluidized bed, where he used the MPT method to determine the vertical distribution of the tracer in a down-scaled cold unit, ${ }^{27,28}$ and Zhang et al. used MPT to measure the particle trajectory in a rotating drum. ${ }^{29}$ The far field of a magnet is uniquely determined by the magnet's position and orientation. Therefore, the field strength at multiple locations is sufficient to relocate the magnet and its orientation. Consequently, the translational and rotational motions of the magnetic particle can be calculated simultaneously. Compared with other noninvasive techniques, the MPT only needs a few magnetometers and a magnetic bead. Therefore, the cost is significantly lower than the RPT, PEPT, and optical-based tracking techniques. In addition, the MPT setup poses no hazardous radiation threat. The advantages and disadvantages of prior techniques are summarized in Table I.

However, the state-of-the-art MPT technology has a severe limitation; its reconstruction process is too slow. The reconstruction is an optimization process of finding the magnet's position and orientation. Hu et al. used the Levenberg-Marquardt method. ${ }^{23}$ Later, Buist and his group identified the sequential quadratic programming (SQP) as the best optimization algorithm..$^{20,30,31}$ However, the cost function in the optimization is highly nonlinear and possesses many local minima. Hence, the reconstruction, which aims to find the global minimum, is time consuming.

In this paper, we develop two new reconstruction methods using the extended Kalman filter $\left(\mathrm{EKF}^{32,33}\right)$ and Sequential Importance sampling with Resampling $\left(\mathrm{SIR}^{34,35}\right)$. These two methods are based on the reformulation of the MPT as a state space model, which is widely used in science and engineering to identify the state of a system from an uncertain model supplemented by a stream of noisy data. Because their performances depend on the specific problem, we test both of them and compare with the SQP method to identify the best approach. In the state space model, the state of the system is regarded as a stochastic process, and its conditional probability distribution based on the given data is approximated. The conditional mean will give the minimum mean square error estimation of the state, and the uncertainty is given by the covariance. If the model and the observation function are both linear and the noises (in the model and observation) are Gaussian, the conditional mean and covariance can be calculated by the Kalman filter. ${ }^{36,37}$ The EKF is an extension of the Kalman filter. It is capable of handling complex dynamics and nonlinear observation functions, but the noises in both model and observation are still assumed to be Gaussian.
The SIR is one of the particle filters, ${ }^{34,35,38}$ which can be used for general nonlinear non-Gaussian situations. We compare the results obtained using the EKF and SIR with those from the SQP. The numerical experiments show that the EKF provides the same accuracy as the SQP, but it is orders of magnitude faster. The efficiency of the SIR falls between the EKF and SQP. The organization of this article is as follows: In Sec. II, the field of a magnetic dipole and the proposed reconstruction methods are discussed. In Sec. III, we discuss using a synthetic magnetic motion to test the SQP, SIR, and EKF algorithms and the uncertainties of these methods are compared. Section IV shows a validation experiment that evaluates the uncertainty of the MPT method. Finally, in Sec. V, the MPT is applied to study the kinetics of one particle in a dense granular shear flow.

\section{COMPUTATIONAL ALGORITHM}

The MPT method uses a small magnet as the excitation source. Far away from the magnet, the field is modeled as a dipole field given by the following equation:

$$
\boldsymbol{B}\left(\boldsymbol{r}, \boldsymbol{r}_{0}, \boldsymbol{m}\right)=\frac{\mu_{0}}{4 \pi} \frac{[3 \boldsymbol{n} \cdot(\boldsymbol{m} \cdot \boldsymbol{n})-\boldsymbol{m}]}{\left|\boldsymbol{r}-\boldsymbol{r}_{0}\right|^{3}},
$$

where $\boldsymbol{r}$ is the magnet's location, $\boldsymbol{r}_{0}$ is the location of a magnet sensor, $\boldsymbol{n}$ is the normal vector in the $\boldsymbol{r}-\boldsymbol{r}_{0}$ direction, $\boldsymbol{m}$ is the magnetic moment, and $\mu_{0}$ is the magnetic permeability. Figure 1 is an

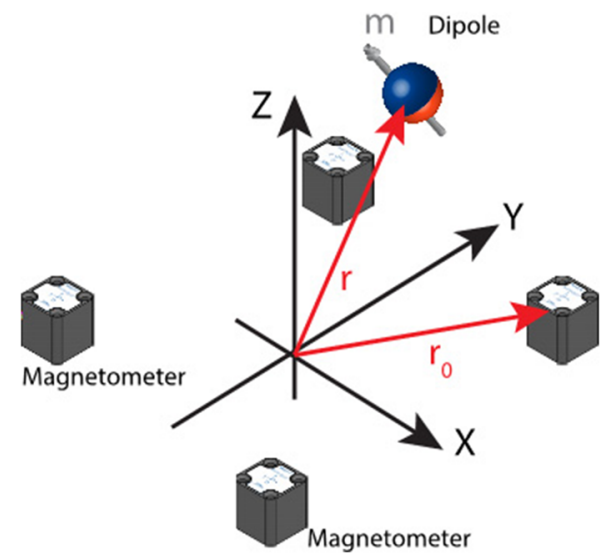

FIG. 1. An illustration of the coordinate system of the MPT. The magnet ball translates and rotates in a certain domain. The cubes stand for an array of magnetometers. 
illustration of the coordinate and measurement setup. Since a magnetometer usually measures the field component in a particular direction $\boldsymbol{S}$, the sensor observation should be $\boldsymbol{B} \cdot \boldsymbol{S}$, i.e., the data from the i-th sensor should be

$$
D_{i}=\boldsymbol{B}\left(\boldsymbol{r}, \boldsymbol{r}_{0 i}, \boldsymbol{m}\right) \cdot \boldsymbol{S}_{\boldsymbol{i}},
$$

where $i=1,2,3, \ldots, \mathrm{N}$ and $\mathrm{N}$ is the total number of sensors. Note that this equation is highly nonlinear. The real observation $O$ from a sensor contains measurement noise and is generally different from this $D$.

Since the position and direction of the sensor can be calibrated accurately, $\boldsymbol{r}_{0 i}$ and $\boldsymbol{S}_{\boldsymbol{i}}$ are known. The task of MPT is to solve Eq. (2) and determine the unknown variables $\boldsymbol{r}$ and $\boldsymbol{m}$ using a set of measured signals. The total degree of freedom (DOF) for the magnet is 6. Nevertheless, if the total magnetic moment of the ball is measured in advance, the DOF reduces to 5. Therefore, at least five independent signals are needed to determine the magnetic field. In reality, the strength of a magnetic field decays as $\left|\boldsymbol{r}-\boldsymbol{r}_{0}\right|^{-3}$; hence, the signal will be very weak if the magnet is far from the sensor. To ensure that the signal-to-noise ratio is high enough, in the following simulations and experiments, we use an array of four 3-axis probes to cover the entire measurement domain (Fig. 1). The total number of signal channels is 12 . For an arbitrary sensor arrangement, Eq. (2) has no simple analytical solution. Therefore, the task becomes an optimization problem, i.e., to find the proper $\boldsymbol{r}$ and $\boldsymbol{m}$ to minimize the following cost function:

$$
J(\boldsymbol{r}, \boldsymbol{m})=\Sigma_{i=1}^{N}\left|O_{i}-\boldsymbol{B}\left(\boldsymbol{r}, \boldsymbol{r}_{0 i}, \boldsymbol{m}\right) \cdot \boldsymbol{S}_{i}\right|,
$$

where $O_{i}$ is the real observation or measurement from the $i$ th sensor. The SQP is an iterative method for constrained nonlinear optimization. The constraint here is that $m_{x}^{2}+m_{y}^{2}+m_{z}^{2}$ must be a constant. If there are no constraints, the SQP method reduces to the well-known Newton's method that finds the point where the gradient of the cost function vanishes. With a constraint, the SQP algorithm solves a sequence of subproblems that optimize a quadratic model of the cost function. The MATLAB program provides the SQP package. For the MPT application, the optimization begins with a global search for the first step. Subsequently, at step $k, \boldsymbol{r}_{k}$ and $\boldsymbol{m}_{\boldsymbol{k}}$ are calculated by minimizing the cost function in Eq. (3), whereas the results from the previous step, $\boldsymbol{r}_{\boldsymbol{k}-1}$ and $\boldsymbol{m}_{\boldsymbol{k}-1}$, serve as the initial guess for the optimization. Once we know $\boldsymbol{r}$ and $\boldsymbol{m}$ at all time steps, we can reconstruct the whole trajectory.

Alternatively, we can reformulate the MPT problem as a statespace model and use different algorithms for the state-space model to solve the MPT reconstruction problem. Let the state variable $\boldsymbol{X}_{k}=\left(\begin{array}{c}\boldsymbol{r}_{\boldsymbol{k}} \\ \boldsymbol{m}_{\boldsymbol{k}}\end{array}\right)$ be the particle status at time step $k$. Given $\boldsymbol{X}_{\boldsymbol{k}-1}, \boldsymbol{X}_{k}$ can be modeled as follows: The location of the magnetic particle $\boldsymbol{r}_{\boldsymbol{k}}$ depends on its position and velocity at previous step $k-1$ and can be modeled by a kinematic equation,

$$
\boldsymbol{r}_{k}=\boldsymbol{r}_{k-1}+\boldsymbol{u}_{k-1} \Delta t+\boldsymbol{w}_{k}^{r} .
$$

The rotation of the magnetic particle can be calculated using a quaternion $q$ as

$$
\boldsymbol{m}_{k}=\boldsymbol{q}_{k-1} \boldsymbol{m}_{\boldsymbol{k}-1} \boldsymbol{q}_{k-1}^{-1}+\boldsymbol{w}_{k}^{m},
$$

where $\boldsymbol{u}_{k-1}$ is the velocity of the magnet at time $k-1, \Delta t$ is the time interval, $\boldsymbol{w}_{k}^{r}$ and $\boldsymbol{w}_{k}^{m}$ are two independent Gaussian variables, which represent unknown perturbations or random noises in the motion and rotation equations, and $q=\left(\cos \frac{\Omega \Delta t}{2}, \omega_{x} \sin \frac{\Omega \Delta t}{2}\right.$, $\left.\omega_{y} \sin \frac{\Omega \Delta t}{2}, \omega_{z} \sin \frac{\Omega \Delta t}{2}\right) .\left(\omega_{x}, \omega_{y}, \omega_{z}\right)$ is a unit vector along the axis of rotation, and $\Omega$ is the magnitude of angular velocity. $\boldsymbol{u}$ and $q$ can be estimated using historic trajectory data. Therefore, we obtain our forward model of the state variable $\boldsymbol{X}_{\boldsymbol{k}}$ as

$$
\boldsymbol{X}_{k}=\left(\begin{array}{c}
\boldsymbol{r}_{k} \\
\boldsymbol{m}_{k}
\end{array}\right)=F\left(\boldsymbol{X}_{\boldsymbol{k}-1}\right)+\boldsymbol{w}_{k},
$$

where

$$
\boldsymbol{F}\left(\boldsymbol{X}_{\boldsymbol{k - 1}}\right)=\left(\begin{array}{c}
\boldsymbol{r}_{k-1}+\boldsymbol{u}_{k-1} \Delta t \\
\boldsymbol{q}_{k-1} \boldsymbol{m}_{\boldsymbol{k - 1}} \boldsymbol{q}_{k-1}^{-1}
\end{array}\right)
$$

and

$$
\boldsymbol{w}_{\boldsymbol{k}}=\left(\begin{array}{c}
\boldsymbol{w}_{k}^{r} \\
\boldsymbol{w}_{k}^{m}
\end{array}\right) .
$$

We assume that the mean of $\boldsymbol{w}_{\boldsymbol{k}}$ is zero and the covariance matrix is $Q_{k}$. The observation (measurement) equation for the $j$ th sensor at time step $k$ is

$$
O_{j, k}=D_{j}\left(\boldsymbol{X}_{k}\right)\left(1+\boldsymbol{v}_{j, k}\right),
$$

where

$$
D\left(\boldsymbol{X}_{k}\right)=\left(\begin{array}{c}
D_{1}\left(\boldsymbol{X}_{k}\right)=B\left(\boldsymbol{X}_{k}\right) \cdot S_{1} \\
\vdots \\
D_{j}\left(\boldsymbol{X}_{k}\right)=B\left(\boldsymbol{X}_{k}\right) \cdot S_{j} \\
\vdots \\
D_{N}\left(\boldsymbol{X}_{k}\right)=B\left(\boldsymbol{X}_{k}\right) \cdot S_{N}
\end{array}\right),
$$

and $\boldsymbol{v}_{k}$ is a vector of Gaussian random variables, which are independent of $\boldsymbol{w}_{k}$ and represent the measurement uncertainties. We assume that $v_{k}$ has a zero mean and the covariance matrix is $R_{k}$.

Equations (4) and (5) together form a state-space model. The task of MPT reconstruction is equivalent to estimating the state variable $\boldsymbol{X}_{k}$ based on the measurements from time step 1 to $k O_{1: k}$. Due to the nonlinearity of our model, we will estimate $\boldsymbol{X}_{k}$ sequentially using EKF and SIR methods. In both cases, one needs to work on the conditional probability distribution of the state $\boldsymbol{X}_{k}$ given the measurement $O_{1: k}$, denoted by $p\left(\boldsymbol{X}_{k} \mid O_{1: k}\right)$. The mean of $p\left(\boldsymbol{X}_{k} \mid O_{1: k}\right)$ will give the minimum mean square error estimate of the state $\boldsymbol{X}_{k}$, and the uncertainty is given by the covariance of $p\left(\boldsymbol{X}_{k} \mid O_{1: k}\right)$.

In the EKF, $p\left(\boldsymbol{X}_{k} \mid O_{1: k}\right)$ is approximated by a Gaussian distribution, which can be completely characterized by the mean and covariance matrix. We assume that the mean at time step $k-1$ is $\boldsymbol{X}_{k-1}$ and the covariance is $P_{k-1}$. Subsequently, the mean $\boldsymbol{X}_{k}$ and the covariance $P_{k}$ are obtained in two steps: prediction and analysis.

(a) In the prediction step, the predicted mean of the trajectory $\boldsymbol{X}_{k}^{-}$ is obtained using the model equation (4),

$$
\boldsymbol{X}_{k}^{-}=\left(\begin{array}{c}
r_{k}^{-} \\
m_{k}^{-}
\end{array}\right)=F\left(\boldsymbol{X}_{k-1}\right)=\left(\begin{array}{l}
\boldsymbol{r}_{k-1}+\boldsymbol{u}_{k-1} \Delta t \\
\boldsymbol{q}_{k-1} \boldsymbol{m}_{k-1} \boldsymbol{q}_{k-1}^{-1}
\end{array}\right) .
$$

The covariance matrix $P_{k-1}$ is updated to $P_{k}^{-}$as

$$
P_{k}^{-}=F_{k} P_{k-1} F_{k}^{T}+Q_{k}
$$


where $F_{k}=\frac{\partial F}{\partial X}\left(\boldsymbol{X}_{\boldsymbol{k}-1}\right)$ is the Jacobian of the model function $F$ evaluated at $\boldsymbol{X}_{\boldsymbol{k}-1}$.

(b) In the analysis step, $O_{k}$, the measurement at time step $k$, is used to update $\boldsymbol{X}_{k}^{-}$and $P_{k}^{-}$by the Kalman formula as follows:

$$
\begin{gathered}
\boldsymbol{X}_{k}=\boldsymbol{X}_{k}^{-}+K_{k}\left(O_{k}-D\left(\boldsymbol{X}_{k}^{-}\right)\right), \\
P_{k}=\left(I-K_{k} H_{k}\right) P_{k}^{-},
\end{gathered}
$$

where the matrix $K_{k}$ is the standard EKF correction matrix as

$$
K_{k}=P_{k}^{-} H_{k}^{T}\left(H_{k} P_{k}^{-} H_{k}^{T}+V_{k} R_{k} V_{k}^{T}\right)^{-1} .
$$

Here, $H_{k}=\frac{\partial D}{\partial X}\left(\boldsymbol{X}_{k}^{-}\right)$is the Jacobian matrix of the measurement function $D$ evaluated at $\boldsymbol{X}_{k}^{-}$and $V_{k}=\frac{\partial O_{k}}{\partial v_{k}}$ is the Jacobian of the observation with respect to $\boldsymbol{v}_{k}$.

In the SIR method, instead of the mean and covariance matrix, the conditional (posterior) probability distribution of the state variables of the whole trajectory $p\left(\boldsymbol{X}_{0: k} \mid O_{1: k}\right)$ is presented by a set of samples with associated weights. Suppose at time step $k-1$, we have presented $p\left(\boldsymbol{X}_{0: k-1} \mid O_{1: k-1}\right)$ by a set of samples $\left\{\boldsymbol{X}_{0: k-1}^{i}\right\}_{i=1}^{N_{s}}$ with the associated weights $\left\{\omega_{0: k-1}^{i}\right\}_{i=1}^{N_{s}}$, where $N_{s}$ is the number of virtual samples. In this paper, 5000 samples are used in the MPT reconstruction. At time step $k$, we first use the model equation (4) to propagate the samples to step $k$ as $i=1, \ldots, N_{s}$,

$$
X_{k}^{i}=\left(\begin{array}{c}
r_{k}^{i} \\
m_{k}^{i}
\end{array}\right)=F\left(X_{k-1}^{i}\right)+w_{k}^{i}
$$

where $w_{k}^{i}$ is a realization of the Gaussian variable $w_{k}$ with a zero mean and a covariance $Q_{k}$.

The observation equation (5) is used to calculate the likelihood $P\left(O_{k} \mid X_{k}^{i}\right)$, and the weight for each virtual sample is updated as

$$
\omega_{k}^{i}=\omega_{k-1}^{i} P\left(O_{k} \mid X_{k}^{i}\right) .
$$

Finally, these weights are normalized as their sum should be 1 . If the variance of the weights is too large, resampling is used to make the weight uniform (see algorithm 3 in Ref. 34 for details).

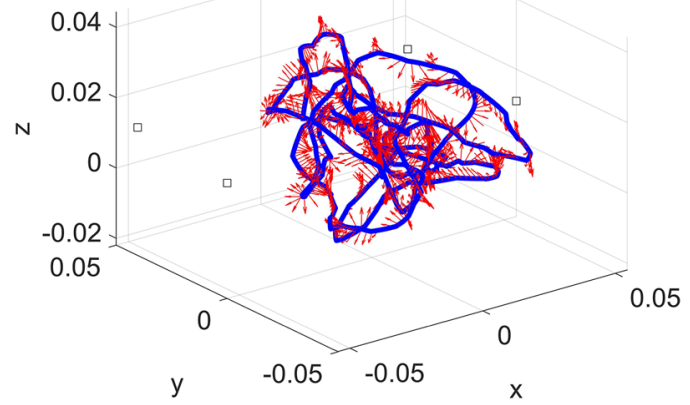

FIG. 2. The blue path is the synthetic trajectory of a magnetic ball, and the red arrows represent the orientation of the magnet. The square boxes represent the magnetometers in the simulations. The unit of the axis is meter.
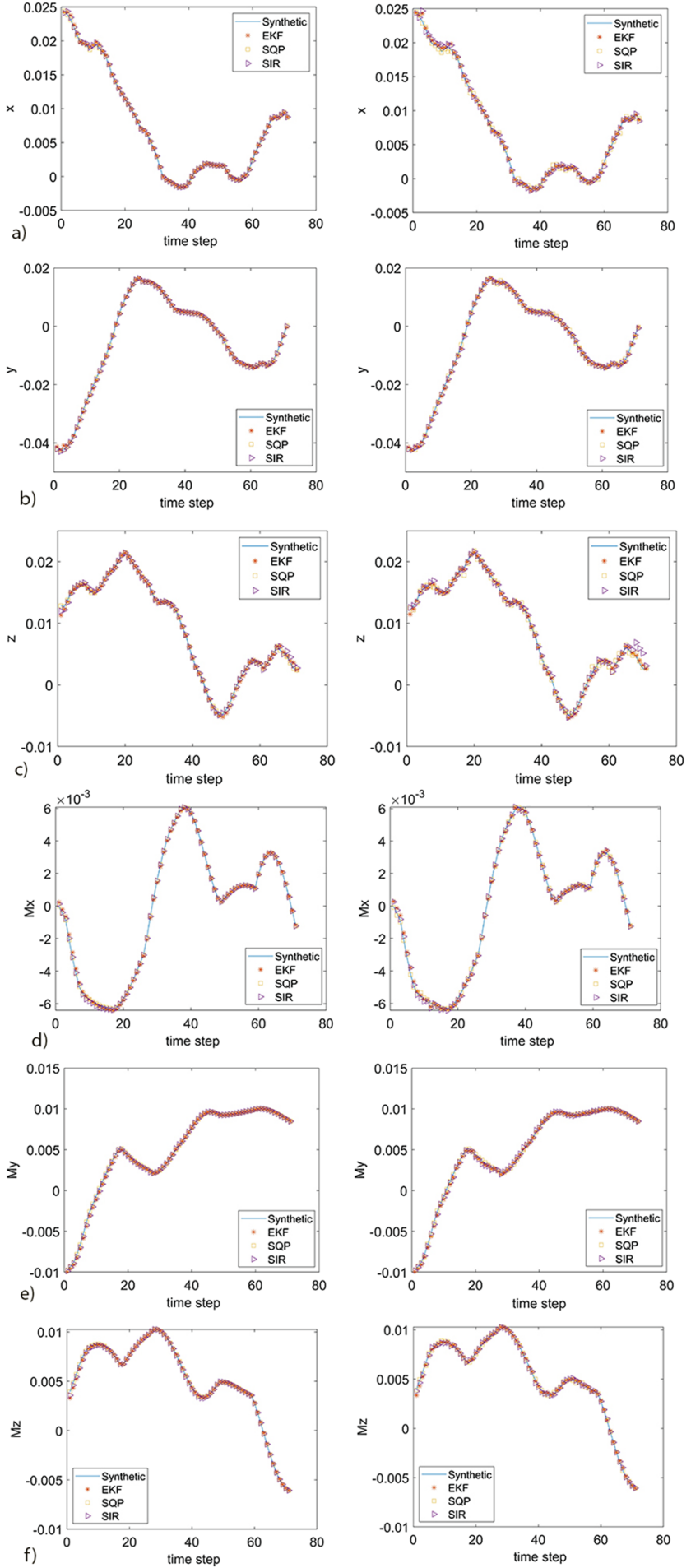

FIG. 3. Sample trajectory sections reconstructed using the SQP, SIR, and EKF with no noise (the left column) and $3 \%$ added noise (the right column). (a), (b), and (c) are for $x, y$, and $z$ component, respectively; (d), (e), and (f) are for $M_{x}, M_{y}$, and $M_{z}$, respectively. The unit of $x, y$, and $z$ is meter and that of $M_{x}, M_{y}$, and $M_{z}$ is $\mathrm{Am}^{2}$. 
TABLE II. The position and rotation error vs the level of noise.

\begin{tabular}{lccccccc}
\hline \hline Error & Method & No noise & $1 \%$ & $3 \%$ & $6 \%$ & $10 \%$ & $20 \%$ \\
\hline \multirow{3}{*}{ Position error (\%) } & SQP & 0.003 & 0.21 & 0.6 & 1.1 & 1.49 & 8.41 \\
& EKF & 0.24 & 0.3 & 0.52 & 0.94 & 1.51 & 9.88 \\
& SIR $^{\mathrm{a}}$ & 0.41 & 0.5 & 0.86 & 1.56 & $\ldots$ & $\ldots$ \\
\hline \multirow{5}{*}{ Rotation error (deg) } & SQP & 0.053 & 0.30 & 0.83 & 1.61 & 2.6 & 5.73 \\
& EKF & 0.76 & 0.81 & 1.10 & 1.78 & 2.7 & 5.54 \\
& SIR $^{\mathrm{a}}$ & 1.43 & 1.43 & 1.74 & 2.77 & $\ldots$ & $\ldots$ \\
\hline \hline
\end{tabular}

${ }^{\mathrm{a}}$ In the SIR computation, 5000 samples were used.

The estimation of $\boldsymbol{X}_{k}$ at step $k$ is approximated by the weighted mean of the samples, i.e., $\boldsymbol{X}_{k}=\sum_{i=1}^{N_{s}} \omega_{k}^{i} \boldsymbol{X}_{k}^{i}$. The uncertainty is approximated by the weighted sample covariance. In both the EKF and SIR, we only need to solve the optimization problem [Eq. (3)] at the beginning of a trajectory to obtain the initial state of the system.

\section{PERFORMANCES OF ALL RECONSTRUCTION ALGORITHMS}

We use several simulations to evaluate the performances of the SQP, SIR, and EKF methods in the reconstruction of trajectories. Not to lose generality, a synthetic trajectory is created by randomly a)

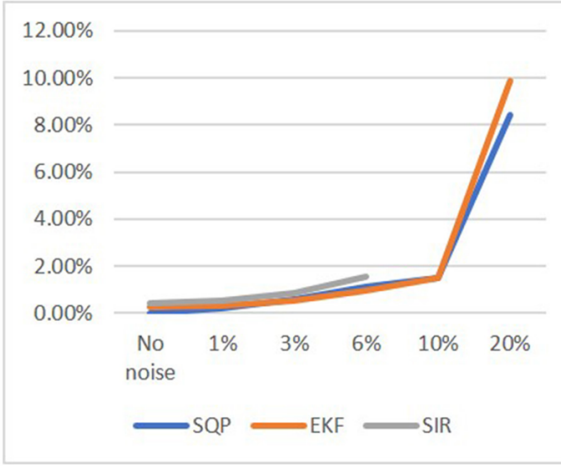

b)

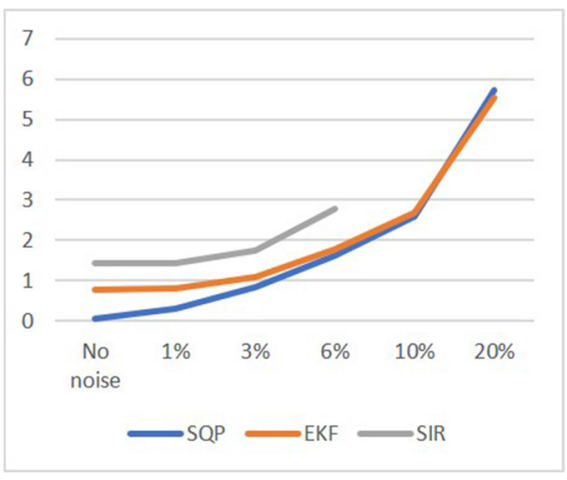

FIG. 4. (a) Position error dependence on the noise and (b) the rotation error vs the noise level.

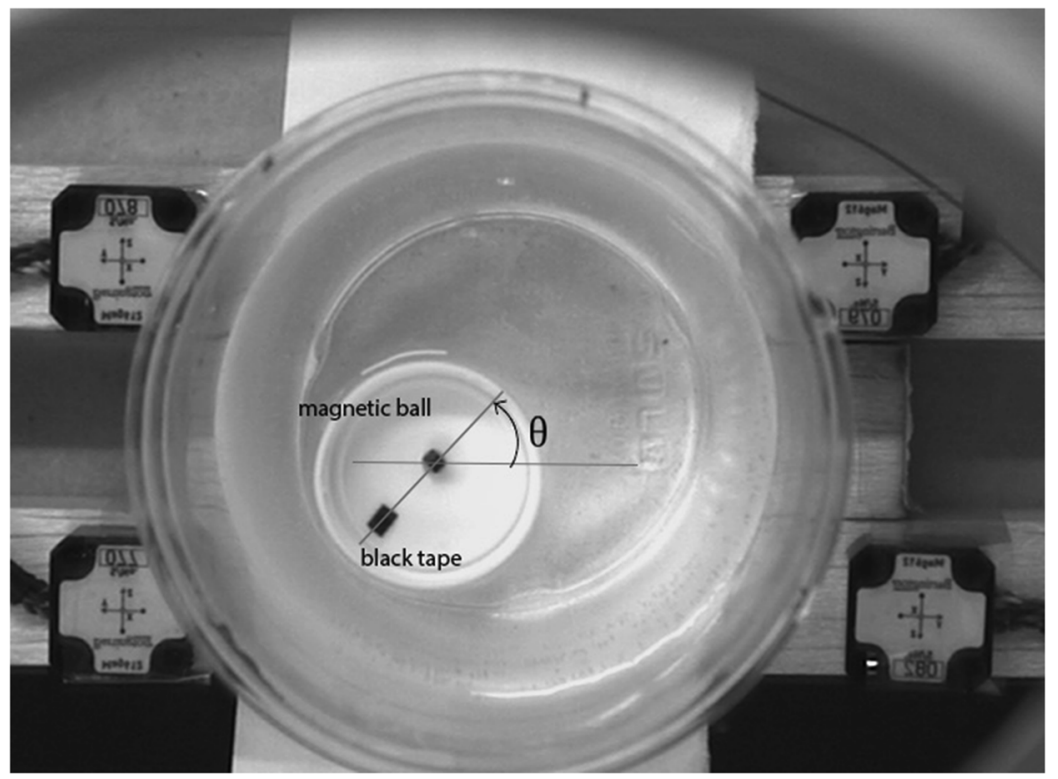

FIG. 5. A top view of the validation experiment. A cap is floating on the water contained in a plastic bottle. The magnetic ball is glued to the cap center. A black marker near the edge of the cap is used to indicate the orientation of the magnet. Four 3-axis magnetometers are placed near the measurement domain. 
perturbing the motion of an object so that the velocity becomes discontinuous along the trajectory (Fig. 2). The magnet moment is set to be $0.0105 \mathrm{Am}^{2}$. A real magnet with this moment is used in the following experiments. The blue curve indicates the location of the magnet, and the red quivers represent the magnet moment. To evaluate the robustness of SQP, SIR, and EKF at multiple noise levels, Gaussian noise is added to the synthetic sensor readings. Specifically, for the $i$ th sensor at each time step, $O_{i}$ is set to be $\boldsymbol{B}\left(\boldsymbol{r}, \boldsymbol{r}_{0 i}, \boldsymbol{m}\right) \cdot \boldsymbol{S}_{\boldsymbol{i}}$. $(1+\epsilon)$, where $\epsilon$ has a normal distribution with a zero mean. The standard deviation of $\epsilon$ is selected to be $0.01,0.03,0.06,0.1$, and 0.2 in different simulations. Multiple trajectories are reconstructed based on the data with these noise levels. Figure 3 shows a comparison of the synthetic and reconstructed trajectories. The total time step in the synthetic trajectory is 5000 , but only a section (with no noise and $3 \%$ added noise) is presented for clarity.

To set a standard in estimating the accuracy of MPT reconstruction, we define the relative error as the difference between the real and reconstructed positions divided by the domain size. For the $x$ component, $E_{x}=\operatorname{mean}\left(\left|x_{j}^{*}-x_{j}\right|\right) / L_{x}$. Here, $L_{x}$ is the size of the domain in the $x$ direction, $L_{x}=[\max (x)-\min (x)], x_{j}^{*}$ is the real value of the position. In the simulation, it is the position of the synthetic trajectory. Similarly, we can calculate the errors in the $y$ and $z$ directions. Consequently, the average relative position error of all components is $\frac{1}{3}\left(E_{x}+E_{y}+E_{z}\right)$. The orientation error can be measured using the mean angle between the measured vector $\boldsymbol{m}$ and the real $\boldsymbol{m}^{*}$.

Table II shows the uncertainties in the position and direction obtained from the above simulations, and the same results are plotted in Fig. 4. We notice that the SIR may not return a useful value when the noise level is too high. The noise level of a typical magnetometer is smaller than $5 \%$. Taking the $3 \%$ noise case as an example, the relative position error of EKF results is $0.52 \%$, better than the SQP and SIR. In the lower noise cases, the SQP is slightly better. With $3 \%$ noise, the angular accuracy of MPT is in the order of $1^{\circ}$. The SQP orientation result is better than the other two approaches. We can compare the position errors with that of optical methods. The image-based position uncertainty can be estimated as 1 pixel, and the field of view is typically $1 \mathrm{~K} \times 1 \mathrm{~K}$. Hence, the relative error is in the order of $0.1 \%$. Therefore, the accuracy of MPT position reconstruction is not very far from that of optical methods.

Despite the above methods having similar robustness against noise, their processing speeds differ significantly. The SIR takes about one third the time of the SQP; and the EKF is about 1000 times faster than the SQP. The reason is that the SQP solves multiple suboptimization problems without the guidance of a model equation, i.e., it breaks one time step into multiple small steps. By contrast, the EKF directly estimates the most probable position of one particle in one step. As for the SIR method, since it generates and follows thousands of virtual samples, its speed is much slower than the EKF. Considering both accuracy and speed, the most costeffective method is the EKF. Therefore, we use it to process our data in the following experiments.

\section{EXPERIMENTAL EVALUATION OF RECONSTRUCTION NOISE}

A floating magnet is used to experimentally evaluate the MPT uncertainty. The magnetic particle is made of neodymium rare earth with a diameter of $3 \mathrm{~mm}$. As shown in Fig. 5, a plastic bottle cap containing the magnetic ball is floating on the surface of water. A black marker is used to indicate the orientation of the magnet (the angle $\theta$ ). The cap is driven by random disturbances. In order to obtain an independent measurement of the motion, a high-speed camera is used to record the magnet's trajectory from the top at a
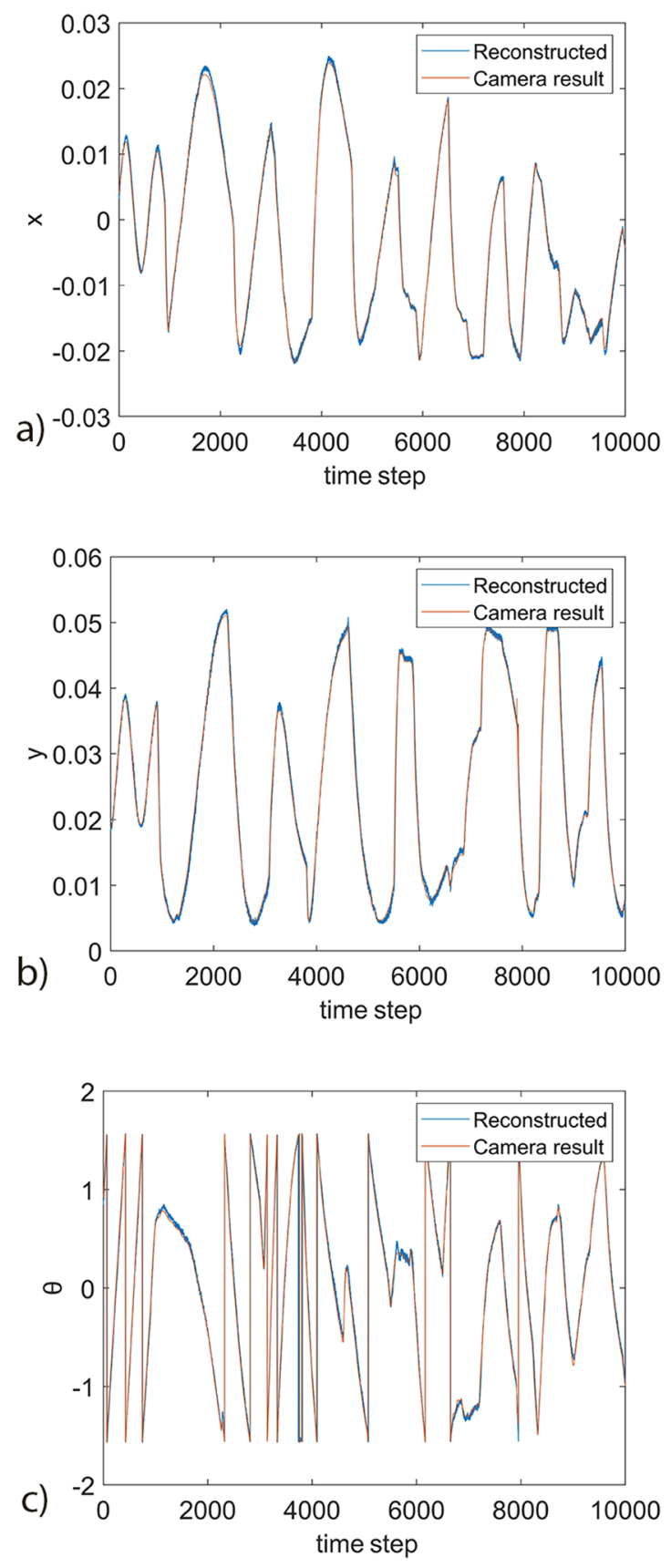

FIG. 6. The comparison between the reconstructed trajectory and the result from a camera for (a) the $x$ component, (b) the y component, and (c) the rotation angle. The units in (a) and (b) are meter, and $\theta$ is measured in radian. 
frame rate of 1000 . We can identify the magnet position and orientation through processing the images. Four Bartington M612 probes (three-axis fluxgate magnetometers) are distributed around the measurement domain. Their sensing range is $[-90 \mu \mathrm{T}, 90 \mu \mathrm{T}]$. The sampling frequency is set to be $1000 \mathrm{~Hz}$ and synchronized with the camera. The coordinates of each sensor are precisely determined by an accurately machined holder. At the beginning of each experiment, the empty domain without any magnet is measured as the offset. Thereafter, the magnetic ball is introduced into the domain, where the sensor array measures the magnetic field change. All devices are fixed on a wooden table, and ferromagnetic materials are kept away from the workbench. The noise level is about $3 \%$ of the measured value.

Figure 6 shows that the MPT reconstructed trajectory and the camera-based results almost overlap. Note that the range of rotation angle is $-\pi / 2$ to $\pi / 2$, so there are sudden jumps between the upper and lower limits in the $\theta$ trajectory. Since the imagebased results have higher accuracy, we use them as the ground truth to evaluate the uncertainty of the MPT with EKF reconstruction. The average relative position error $\frac{1}{2}\left(E_{x}+E_{y}\right)$ is $0.65 \%$, and the error in $\theta$ determination is $1.44^{\circ}$. These results show that the MPT method successfully captures the motion of the tracer particle, which is consistent with the simulation results discussed in Sec. III.

\section{APPLICATION OF MPT IN A GRANULAR SHEAR FLOW}

Granular materials are widely used in industry, but granular flow is far from being understood. This is partially because of technical difficulties in measuring this type of flow. To demonstrate the usefulness of MPT, we applied it to investigate a granular shear flow. We generate this flow in a cylindrical container with a rotating disk at the bottom, as shown in Fig. 7(a). The rotating disk is connected by a shaft to a DC motor that is located $0.5 \mathrm{~m}$ away from the measurement domain. Such a distance is sufficient to prevent the motor magnetic field from interfering with the MPT measurements. In addition, there is no ferromagnetic material near the magnetometer. The inner diameter of the cylindrical container is $101 \mathrm{~mm}$, and the rotating disk diameter is $63.5 \mathrm{~mm}$. There are 385 brass balls randomly packed in the container [Fig. $7(\mathrm{~b})$ ], each with a diameter of $10 \mathrm{~mm}$. One of the balls encapsulates a magnetic bead, which serves as the tracer particle. The weight of this tracer ball is $3.604 \mathrm{~g}$, and that of the normal balls is $3.709 \mathrm{~g}$. Therefore, the tracer has nearly the same mechanical property as the normal balls. The rotation speed of the bottom disk is $10 \mathrm{rpm}$. The Bartington M612 probes are installed on two rings with a distance of $37.5 \mathrm{~mm}$ between them [Fig. 7(a)]. The magnetometers are located $67.5 \mathrm{~mm}$ away from the center of the container.

The experiment procedures have been described in Sec. IV. Specifically, the background magnetic field is the same as that in the validation test, which confirms that the motor has very little effect in the measured domain. The sampling frequency is set to be $50 \mathrm{~Hz}$, which is sufficient to capture the slow motion. Figure 8 displays the reconstructed path using the EKF. Since the grain diameter is large and the driving rotation is only $10 \mathrm{rpm}$, the balls show a layered distribution. The tracer ball, which was initially near the center, is able to reach five layers in the radial direction and 4 layers in the vertical direction (Fig. 8). The probability of staying at each layer is not the

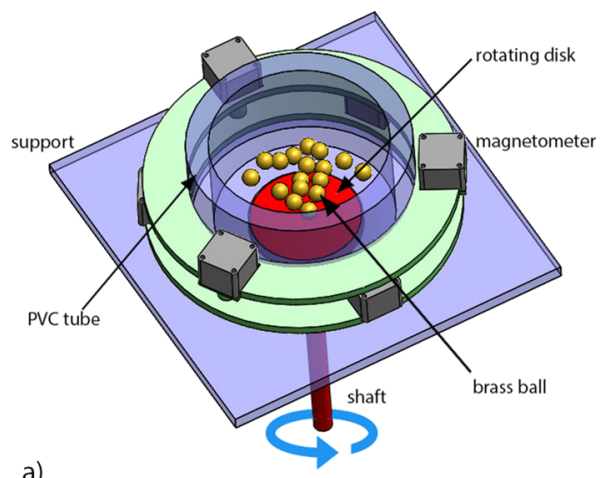

a)

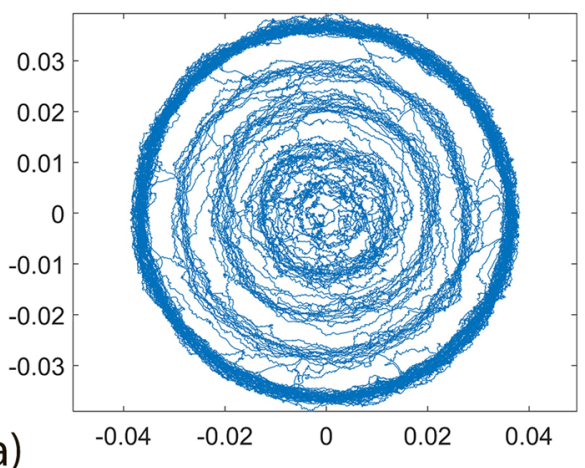

b)
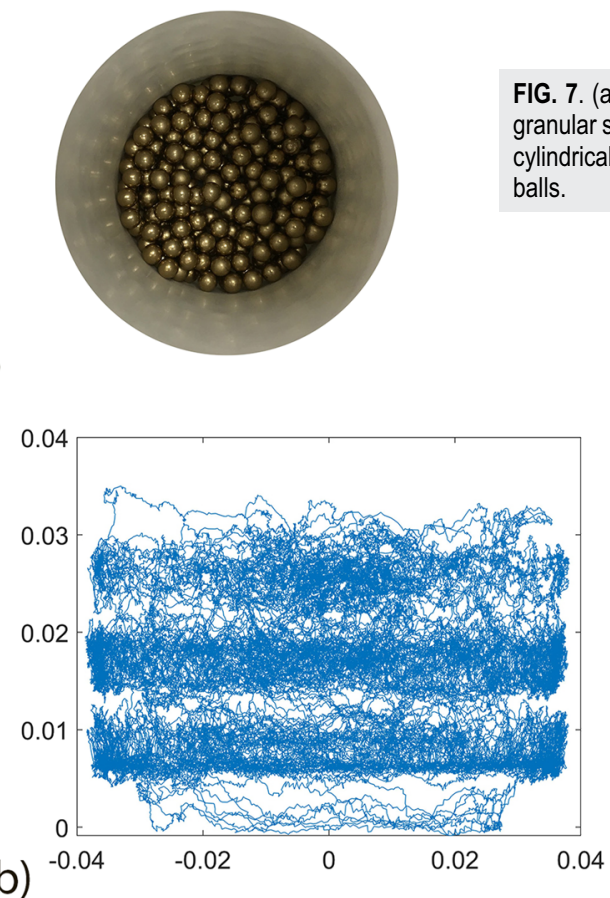

FIG. 7. (a) An illustration of the device used to generate a granular shear flow. (b) The top view of the brass balls in the cylindrical container. The tracer ball is hidden among these balls.
FIG. 8. The top view (a) and side view (b) of the trajectory of a tracer particle in a granular shear flow. The unit is meter. 

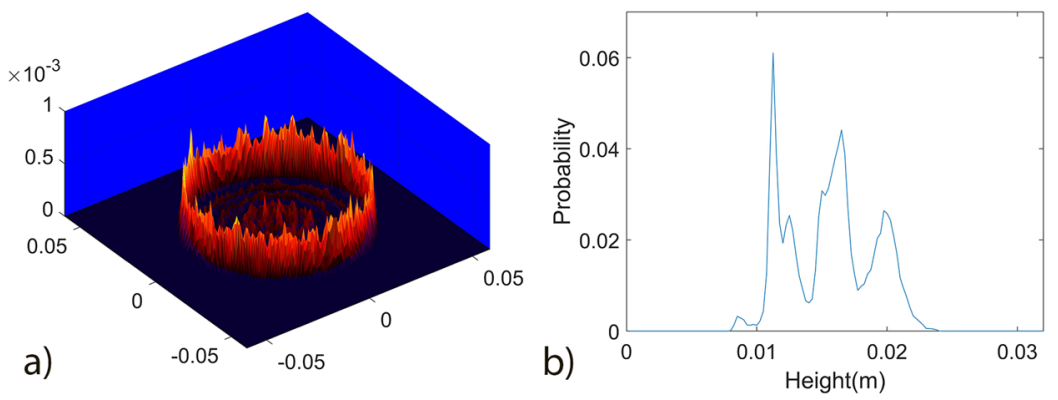

FIG. 9. The probability distribution in the $x-y$ plane (a) and in the vertical direction (b). The unit in (a) is meter. same. We calculated the radial and azimuthal probability distribution. The tracer has a higher chance of staying at the periphery and at the center [Fig. 9(a)]. This periphery diameter is about $70 \mathrm{~mm}$, and there are two more layers of balls between this periphery and the cylinder wall. However, over the investigated time period, the tracer was not able to penetrate into these outer layers. The probability distribution in the vertical direction is shown in Fig. 9(b). The top three vertical layers have significant probability, but the bottom layer has little.

\section{SUMMARY}

In this research, we have developed a tracking system that uses the magnetic field to measure the motion of a particle. A great advantage of the MPT method is that it does not rely on any hazardous radiation such as the $\mathrm{X}$-rays to measure the motion in an opaque environment. The cost is therefore much lower. Another important feature of MPT is that it can simultaneously measure the position and orientation of a particle, whereas the orientation is difficult to obtain using other technologies.

We tested two new algorithms (the SIR and EKF) to reconstruct the MPT trajectory and orientation. Their accuracies are comparable to that of the state-of-the-art optimization approach, but the EKF reconstruction speed is orders of magnitude faster. Specifically, the validation experiment shows that the MPT position uncertainty can reach $0.6 \%$ and the angular error is $1.44^{\circ}$ for a measurement domain with a size of about $10 \mathrm{~cm}$. The position accuracy is lower but comparable with that of advanced image-based methods.

The MPT technology was used to track the motion in a dense granular shear flow. Our results demonstrate that the MPT is able to capture the trajectory of a tracer particle, from which it is possible to obtain the spatial probability distribution in both horizontal and vertical directions. Because of the above advantages and its high accuracy, MPT is a powerful tool for studying dense granular flows.

\section{ACKNOWLEDGMENTS}

The authors would like to thank Dr. Huazhen Fang for helping us develop the Particle filter and Kalman filter for the MPT, and we are grateful to Dr. Kay Buist for discussing with us the SQP algorithm. In addition, we acknowledge Wes Ellison for his help on the hardware and Randi Hacker and John Haug for the preparation of this manuscript.
This project is supported by the University of Kansas General Research Funding.

\section{REFERENCES}

${ }^{1}$ F. Charru, B. Andreotti, and P. Claudin, Annu. Rev. Fluid Mech. 45, 469 (2013).

${ }^{2}$ F. Toschi and E. Bodenschatz, Annu. Rev. Fluid Mech. 41, 375 (2009).

${ }^{3}$ N. Chenouard, I. Smal, F. De Chaumont, M. Maška, I. F. Sbalzarini, Y. Gong, J. Cardinale, C. Carthel, S. Coraluppi, M. Winter, A. R. Cohen, W. J. Godinez, K. Rohr, Y. Kalaidzidis, L. Liang, J. Duncan, H. Shen, Y. Xu, K. E. G. Magnusson, J. Jaldén, H. M. Blau, P. Paul-Gilloteaux, P. Roudot, C. Kervrann, F. Waharte, J. Y. Tinevez, S. L. Shorte, J. Willemse, K. Celler, G. P. Van Wezel, H. W. Dan, Y. S. Tsai, C. O. De Solórzano, J. C. Olivo-Marin, and E. Meijering, Nat. Methods 11, 281 (2014).

${ }^{4}$ A. Amon, P. Born, K. E. Daniels, J. A. Dijksman, K. Huang, D. Parker, M. Schröter, R. Stannarius, and A. Wierschem, Rev. Sci. Instrum. 88, 051701 (2017).

${ }^{5}$ N. T. Ouellette, H. Xu, and E. Bodenschatz, Exp. Fluids 40, 301 (2006).

${ }^{6}$ X. Guo, J. Tang, J. Li, C. Shen, and J. Liu, "Attitude measurement based on imaging ray tracking model and orthographic projection with iteration algorithm," ISA Trans. (in press).

${ }^{7}$ X. Guo, J. Tang, J. Li, C. Wang, C. Shen, and J. Liu, Rev. Sci. Instrum. 90, 033704 (2019).

${ }^{8}$ J. S. Lin, M. M. Chen, and B. T. Chao, AIChE J. 31, 465 (1985).

${ }^{9}$ D. Parker, D. Allen, D. Benton, P. Fowles, P. McNeil, M. Tan, and T. Beynon, Nucl. Instrum. Methods Phys. Res., Sect. A 392, 421 (1997).

${ }^{10}$ D. Parker, R. Forster, P. Fowles, and P. Takhar, Nucl. Instrum. Methods Phys. Res., Sect. A 477, 540 (2002).

${ }^{11}$ D. J. Parker, M. R. Hawkesworth, C. J. Broadbent, P. Fowles, T. D. Fryer, and P. A. McNeil, Nucl. Instrum. Methods Phys. Res., Sect. A 348, 583 (1994).

${ }^{12}$ D. J. Parker, C. J. Broadbent, P. Fowles, M. R. Hawkesworth, and P. McNeil, Nucl. Instrum. Methods Phys. Res., Sect. A 326, 592 (1993).

${ }^{13}$ R. D. Wildman and D. J. Parker, Phys. Rev. Lett. 88, 064301 (2002).

${ }^{14}$ J. B. Romero and D. W. Smith, AIChE J. 11, 595 (1965).

${ }^{15}$ E. W. Grohse, AIChE J. 1, 358 (1955).

${ }^{16}$ X. Chen, W. Zhong, and T. J. Heindel, AIChE J. 65, 520 (2019).

${ }^{17}$ T. J. Heindel, J. Fluids Eng. 133, 074001 (2011).

${ }^{18}$ H. Nadeem and T. J. Heindel, Powder Technol. 332, 331 (2018).

${ }^{19}$ C. Scholz and T. Pöschel, Phys. Rev. Lett. 118, 198003 (2017).

${ }^{20}$ K. A. Buist, T. W. Van Erdewijk, N. G. Deen, and J. A. M. Kuipers, AIChE J. 61, 3198 (2015).

${ }^{21}$ H. Wu, in AIAA Scitech 2019 Forum (AIAA, 2019), p. 272.

${ }^{22}$ W. Andrä, H. Danan, W. Kirmsse, H. H. Kramer, P. Saupe, R. Schmieg, and M. E. Bellemann, Phys. Med. Biol. 45, 3081 (2000).

${ }^{23}$ C. Hu, M. Li, S. Song, W. Yang, R. Zhang, and M. Q. Meng, IEEE Sens. J. 10, 903 (2010).

${ }^{24}$ G. Mohs, O. Gryczka, and S. Heinrich, Chem. Eng. Sci. 64, 4811 (2009).

${ }^{25}$ V. Idakiev and L. Mörl, J. Chem. Technol. Metall. 48, 445 (2013).

${ }^{26}$ E. E. Patterson, J. Halow, and S. Daw, Ind. Eng. Chem. Res. 49, 5037 (2010). 
${ }^{27}$ A. Köhler, D. Pallarès, and F. Johnsson, Fuel Process. Technol. 162, 147 (2017).

${ }^{28}$ A. Köhler, A. Rasch, D. Pallarès, and F. Johnsson, Powder Technol. 316, 492 (2017).

${ }^{29}$ L. Zhang, F. Weigler, V. Idakiev, Z. Jiang, L. Mörl, J. Mellmann, and E. Tsotsas, Powder Technol. 339, 817 (2018).

${ }^{30}$ K. A. Buist, A. C. van der Gaag, N. G. Deen, and J. A. M. Kuipers, AIChE J. 60, 3133 (2014).

${ }^{31}$ K. A. Buist, P. Jayaprakash, J. A. M. Kuipers, N. G. Deen, and J. T. Padding, AIChE J. 63, 5335 (2017).
${ }^{32}$ Y. Shi and H. Fang, Int. J. Control 83, 538 (2010).

${ }^{33}$ F. Gustafsson, Adaptive Filtering and Change Detection (Wiley, New York, 2000).

${ }^{34}$ M. S. Arulampalam, S. Maskell, N. Gordon, and T. Clapp, IEEE Trans. Signal Process. 50, 174 (2002).

${ }^{35}$ A. Smith, Sequential Monte Carlo Methods in Practice (Springer Science \& Business Media, 2013).

${ }^{36}$ A. Gelb, Applied Optimal Estimation (MIT Press, Cambridge, 1974).

${ }^{37}$ R. E. Kalman and R. S. Bucy, J. Basic Eng. 83, 95 (1961).

${ }^{38}$ A. J. Chorin and X. Tu, Proc. Natl. Acad. Sci. U. S. A. 106, 17249 (2009). 